\title{
LA LUCHA CONTRA LA LEPRA
}

EN LA ESPAÑA DE LA PRIMERA MITAD DEL

SIGLO XX. EVOLUCIÓN

DE LAS ESTRATEGIAS PREVENTIVAS

BASADA EN LOS AVANCES CIENTÍFICOS, SANITARIOS Y SOCIALES

\section{Rosa Sánchez García}

\section{Introducción}

El carácter infeccioso de la lepra es ya conocido a principios de siglo: fue en 1873 cuando Hansen descubrió el bacilo productor de la enfermedad; sin embargo tardó en aceptarse masivamente que el único causante de la lepra fuese el bacilo de Hansen. Con el paso de los años esta concepción de enfermedad infectocontagiosa se fue generalizando y de ahí que el aislamiento fuese la principal medida preventiva.

La lucha contra la lepra en España en la primera mitad del presente siglo tuvo por tanto como medida fundamental el aislamiento, si bien se perciben diferentes formas de practicarlo a lo largo de los años. Los descubrimientos científicos permitieron enfocar el problema desde otras perspectivas y añadir al aislamiento, como única medida efectiva, otras fórmulas para combatir la enfermedad.

Tuvo también gran influencia la respuesta social frente a la enfermedad de Hansen. La postura adoptada por la sociedad frente a los enfer- 
mos leprosos fue en general de rechazo, aunque con los grandes avances conseguidos en el tratamiento en los últimos años de la década de los cuarenta se planteaba necesariamente el regreso del enfermo desde su lugar de aislamiento (leprosería) al seno de su grupo social. El propio enfermo veía con esperanza la posibilidad de curarse con los avances conseguidos en todos estos años y decidió en muchos casos acudir al medico para pedir ayuda, cuando antes ocultaba su enfermedad con las dificultades que esto suponía para el control de la lepra.

Las autoridades sanitarias fueron igualmente influyentes en la lucha contra la enfermedad pues fueron ellas las rectoras de las medidas de lucha a tomar. Los profesionales de la medicina recomendaron unas $\mathrm{u}$ otras acciones, pero sólo cuando las autoridades tomaron la iniciativa pudieron ser eficaces. Se requería una labor de conjunto para luchar contra esta enfermedad, no medidas aisladas que sólo podían resolver el problema focalmente. En este sentido se percibe claramente una voluntad política de lucha contra la enfermedad —como bien señala Granjel- (1) Las luchas sanitarias en la España de los últimos lustros del XIX y primeras décadas del XX se centraron especialmente en enfermedades endémicas con gran repercusión social; la lepra es un prototipo de estas enfermedades y, si cabe, la de mayor repercusión social, además con el agravante de ser España uno de los pocos paises europeos donde aún existían focos endémicos. Es lógico que preocupe a las autoridades sanitarias, pero no tanto como para que tomen medidas efectivas hasta bien avanzado el período que estamos estudiando.

Los profesionales de la medicina - dermatólogos y leprólogos, sobre todo- de gran altura científica, son los que continuamente llaman la atención para que se actúe contra la lepra. Las respuestas publicas son de poca importancia durante las primeras décadas del siglo, quizás, porque el número de enfermos es mucho menor que en el caso de otras enfermedades de características similares. Sin embargo, cada vez son más las voces que piden atención al problema e incluso habrá que enfrentarse a un enjuiciamiento internacional en el VI Congreso Internacional de Leprología que se celebraría en Madrid en 1953. El gobierno no tendrá más remedio que intervenir.

El objetivo de este trabajo es analizar las medidas de lucha contra la lepra en España en la primera mitad del siglo XX y su evolución a lo largo de este período. Las fuentes utilizadas son fundamentalmente colecciones de revistas, folletos, tratados y congresos o reuniones de dermatología y leprología. 
2. Medidas fundamentales de lucha contra la lepra y su evolución en estos años

Esquemáticamente pódemos distinguir los siguientes apartados en las medidas de lucha recomendadas:

I. Declaración obligatoria de la enfermedad con objeto de establecer estadísticas fiables sobre la lepra en España.

II. Aislamiento domiciliario: profilaxis individual.

III. Aislamiento sanatorial: profilaxis social.

IV. Otras medidas. Medidas gubernativas.

Casi todos los autores de más prestigio y foros nacionales e internacionales recomiendan tener en cuenta cada uno de estos puntos, si bien con diferentes matices.

En cuanto a la evolución a lo largo de este período podemos considerar la existencia de tres etapas en la lucha contra la lepra. La primera etapa se extiende entre 1900 y 1925-30; la segunda, de 1930 a 1945-46; la tercera es la etapa previa al VI Congreso Internacional de Leprología celebrado en Madrid en 1953. Precisamente cara a este Congreso el Estado Español se preparó para ofrecer una buena imagen a los leprólogos internacionales, y, como las autoridades sanitarias tomaron parte más activa, se consiguieron niveles eficaces de lucha contra la enfermedad, probablemente los más altos de todo el período estudiado.

Entre la primera y la segunda etapa las diferencias son, quizá, un poco artificiosas, y vienen dadas por los avances científicos fundamentalmente como ya hemos dicho. En la primera etapa la profilaxis y la lucha contra la enfermedad van encaminadas a tomar medidas contra el hombre ya leproso; en la segunda se empieza a ver la importancia que tiene en la lucha el control de las personas que han tenido contacto con el enfermo, es decir, los convivientes.

\subsection{Primera etapa: 1900 a $1925-30$}

Los antecedentes más inmediatos e importantes en lo que a medidas de lucha y legislación se refiere se remontan a una normativa de 1878, en que por Real Orden de 7 de enero se dictan unas disposiciones del Gobierno Español para atajar la propagación de la lepra, se conoce también 
como ley de Romero Robledo. Esta ley contenía las principales medidas de lucha contra la lepra: Declaración obligatoria, aislamiento sanatorial, aislamiento domiciliario y otras medidas relacionadas con las diferentes concepciones patogénicas vigentes, por ejemplo la recomendación de no consumir determinadas carnes o pescados.

Se trataba de una ley, calificada por la mayoría de los autores españoles más prestigiosos de modélica, pues abarcaba todas las medidas que en ese momento se podían tomar para atajar la enfermedad. De haberse cumplido es muy probable que la lepra hubiese disminuido en su extensión o casi desaparecido de nuestro país.

La declaración obligatoria era reconocida de forma unánime como una medida necesaria y de la que debía partir la lucha. A pesar de estar vigente, las estadísticas de leprosos en España no eran exactas y estaban muy lejos de la realidad. Como ejemplo un censo oficial de 1914 daba la cifra de 898 enfermos, mientras que las estadísticas particulares aportadas por leprólogos de la época hablan de entre 1.500 a 2.000 enfermos de lepra (2).

En cuanto al aislamiento, la mayoría de los autores distinguen las dos modalidades: aislamiento sanatorial o profilaxis social y aislamiento domiciliario o profilaxis individual. En las primeras décadas del siglo la elección entre uno u otro se basaba preferentemente en la condición social del individuo enfermo eludiendo la forma clínica o estadio evolutivo de la enfermedad. González Castellano (1905), importante estudioso de la lepra en la comarca de La Marina de Alicante, es claro al respecto: aislamiento en hospitales para los pobres y vigilancia del aislamiento individual para las clases acomodadas (3). Esta recomendación se basaba en varios condicionantes: la profilaxis domiciliaria sólo se podía llevar a cabo observando el leproso un régimen higiénico severo (ropas y utensilios de uso exclusivo, habitación individual, junto a una limpieza exhaustiva) que dificilmente podían practicar los mendigos y leprósos pobres; en muchos casos sin un hogar donde cobijarse. Pérez Dagnino describe claramente esta situación:

«Los pobres; los verdaderamente necesitados habitan en cuevas, zahurdas, cubiles, privados de todo trato y en el más absoluto abandono; alimentándose con el producto de las escasas limosnas y rodeados de un ambiente de suciedad y porquería que se hace incompatible con la vida». 
Descripciones semejantes encontramos en publicaciones de diversos autores de la época (4).

En 1909 se celebró la II Conferencia Internacional contra la lepra en Bergen (Noruega), J. F. Tello representante español expone la situación de nuestro país:

«El incremento de la lepra puesto de manifiesto por la última estadística, ha decidido a obrar el Gobierno Español y sólo aguarda la celebración de la Conferencia, para tomar y hacer ejecutar las medidas oportunas después de oir la voz de tantos sabios aquí reunidos» (5).

La medida fundamental que se siguió recomendando en esta conferencia fue el aislamiento y como novedad aconsejaban: «Revisar todas las teorías sobre la ețiología y el modo de propagación de la lepra», encaminado a buscar el modo de combatir la enfermedad. En España, a pesar de la buena disposición, no se ponen en práctica ni estas medidas ni las establecidas con anterioridad.

Los estudiosos de la lepra reclaman una y otra vez atención al problema pero sólo se detectan medidas aisladas en algunos focos coincidiendo con la presencia de algún profesional decidido a combatir el mal. Como medida de profilaxis social o aislamiento sanatorial destaca la fundación del Sanatorio Leprosería de Fontilles en Alicante (1909), de iniciativa privada y coincidiendo con un foco endémico en esa zona; los años demostraron la eficacia de esta institución para atajar la lepra en el foco levantino. En el resto del Estado solamente estaban aislados en leproserías u hospitales 170 enfermos, según los datos oficiales de 1914 (6), los demás leprosos estaban fuera de todo control.

En años posteriores la situación sigue siendo similar, las medidas recomendadas para combatir la enfermedad son básicamente las mismas, como novedad destaca el estudio que realiza el dermatólogo Sánchez Covisa en el que clasifica los enfermos en tres categorías y según a la que pertenezcan se adoptarán unas $u$ otras medidas de aislamiento: $1 .^{\circ}$ Leprosos incipientes: se podrán tratar en su domicilio, pero es obligatorio el tratamiento y que cumplan unos preceptos higiénicos. $2 .^{\circ}$ Leprosos esterilizados bacteriológicamente, con lesiones cerradas, o no peligrosos. También se podrán tratar en sus domicilios siguiendo las indicaciones de las autoridades sanitarias. Si no cumpliesen estas normas serán forzosamente recluidos. $3 .^{\circ}$ Enfermos peligrosos, con lesiones abiertas, que 
eliminan bacilos por sus secrecciones y por sus heridas: serán forzosamente recluidos por el peligro que suponen para las personas que le rodean (7).

Es interesante destacar la innovación que supuso el tomar en consideración de manera primordial la forma clínica sobre la base de los conocimientos que se iban adquiriendo, antes de adoptar otro tipo de medidas, en relación con situaciones anteriores en que prevalecía la condición social del enfermo.

\subsection{Segunda etapa: 1930 a $1945-46$}

Como hemos dicho anteriormente, este período, no claramente diferenciado del anterior, ofrece una novedad en la lucha contra la lepra. Es algo que ya había sido apuntado por autores anteriores y en Congresos Internacionales, pero es en esos años cuando adquiere interés primordial. Se trata de la vigilancia de las personas que han estado en contacto con los leprosos, es decir, de los convivientes. Ademas, progresivamente, el aislamiento sanatorial va perdiendo importancia en favor de otras medidas como son: el aislamiento individual, el diagnóstico precoz, etc... Los avances científicos permiten afrontar el problema de la lepra desde otras perspectivas; diagnosticar precozmente la enfermedad permite tomar otro tipo de medidas más eficaces.

Las autoridades sanitarias establecieron importantes medidas de lucha en un período en el que destacaron dos hechos de gran transcendencia: el establecimiento de la II República y la Guerra Civil. En la primera se implantaron las bases para una lucha que prometía ser eficaz y que no llegaron a cuajar por el estallido de la Guerra Civil.

A nivel internacional destacaron las conclusiones de la Conferencia Internacional de la lepra celebrada en Bangkok en 1930 y las propuestas de la Comisión de la lepra de la Sociedad de las Naciones reunida en abril de 1931. En ambas, básicamente, se recomendaron las mismas medidas de las cuales destaca el hecho de que recomienden el aislamiento sólo para los casos contagiosos, el considerar necesario que en cada país exista un centro de referencia sobre las investigaciones y conocimientos sobre la lepra, control de los sospechosos, etc... Se considera la lucha contra la lepra como un todo integrado por medidas médicas, legislativas y sociales, no como algo aislado. La parte preventiva tiene gran importancia, así como el control de los sospechosos a que antes hemos aludido. 
Otro dermatólogo, Bejarano (1929), además de insistir en lo que veíamos en el período anterior sobre el diferente comportamiento según la forma clínica de que se tratase, recomendaba las medidas que proponen los grandes leprólogos internacionales Rogers y Muir; son semejantes a las aconsejadas por la Conferencia Internacional de 1930, si bien con algunos puntos más concretos, como el hecho de recomendar que exista una comisión medica que realice el reconocimiento de los casos denunciados, esta comisión estará formada por leprólogos con adecuada capacidad científica.

Otro punto que recomienda Bejarano es el examen periódico cada tres o seis meses y durante tres años de los convivientes con leprosos (8).

Se insistía una vez más en medidas ya conocidas y se daba gran importancia al control de los convivientes con leprosos.

Para completar este abanico de normas y medidas en nuestro país, por orden ministerial de 2 de septiembre de 1933 el Gobierno decretó una legislación sobre lepra. En ese momento era Director General de Sanidad el eminente dermatólogo Bejarano, anteriormente citado: no dudamos de que su influencia fue decisiva para poner estas bases administrativas de lucha contra la lepra. Para el Gobierno era importante dictar una nueva ley para coordinar las medidas desde todos los ámbitos, tal y como se recomendó en la reunión de la Comisión de la Lepra de la Sociedad de las Naciones; las intenciones de esta ley así lo evidenciaban:

"La necesidad de intensificar la lucha contra la lepra y de coordinar los esfuerzos, hoy dispersos, encaminados a tal fin, obligan a dictar normas en consonancia con los conocimientos científicos actuales y con los medios hoy disponibles (9)».

Constaba esta ley de 26 artículos, de los que los más importantes resumidamente serían:

1. Se refiere a la Declaración Obligatoria por parte de los médicos. Los inspectores provinciales de Sanidad velarán porque se cumpla esta norma y facilitarán el material necesario para un diagnóstico exacto.

2. ${ }^{\circ}$ La Inspección Provincial de Sanidad delegará en los médicos de la Organización Nacional Antivenérea para que se encarguen de controlar a los enfermos no hospitalizados; los pobres recibirán tratamiento gratuito. 


\section{(...)}

4. ${ }^{\circ}$ A efectos de aislamiento los enfermos se clasifican en dos grupos: a) formas cerradas que no eliminan bacilos por lesiones ni por moco nasal: podrán permanecer en su domicilio sometidos a vigilancia periódica. b) Formas abiertas con eliminación de bacilos: serán aislados en leproserías; sólo si el aislamiento domiciliario puede hacerse eficazmente se le permitirá, pero habrá de ser vigilado de cerca por el servicio antivenéreo más próximo.

$(\ldots)$

6. Los enfermos aislados en su domicilio no podrán ejercer profesiones que supongan peligro para los demás.

7. Las condiciones de aislamiento domiciliario serán: dormitorio uso exclusivo, utensilios y ropas de uso exclusivo que serán convenientemente desinfectadas y lavadas separadamente.

8. Los enfermos pobres serán aislados en locales apropiados en la provincia donde sean vecinos, siendo responsable de ellos la Diputación Provincial.

9. En los hospitales habrá estancias exclusivas para leprosos con lesiones avanzadas.

10. ${ }^{\circ}$ Si hay colonias leproserías serán enviados ahí preferentemente.

En nuestra opinión los puntos que trataba esta ley habían sido ya recomendados a nivel internacional y por leprólogos o dermatólogos nacionales. Como novedad destaca el hecho de que se responsabilizase a la Junta Central Antivenérea y a los Dispensarios Antivenéreos del control y asesoramiento en la lucha contra la lepra. En realidad, pretendían aprovechar los dispensarios antivenéreos, que existían distribuidos por toda la geografía española, para detectar y seguir los casos de lepra. La condición social del leproso pasaba a un plano secundario aunque no se podía obviar el contingente de leprosos indigentes, sin hogar, a los que la ley amparaba recomendando su aislamiento y recogida.

Esta ley, así como otras recomendaciones para luchar contra la lepra, permanecían incumplidas. Los autores más destacados del momento recomendaban unas u otras acciones que venían a reiterar lo dicho hasta ahora: el problema era que no se llevaban a la práctica. Montañés, director de la leprosería de Fontilles, planteaba la importancia de realizar un censo de sospechosos o de convivientes (10), medida de indudable valor para controlar a los convivientes pero carente de sentido cuando no se contaba ni siquiera con un censo fiable de los enfermos. En este período 
las estadísticas oficiales no recogían todos los casos de lepra, reflejaban sólo una pequeña parte. De los leprosos censados una minoría estaban aislados en sanatorios y el aislamiento individual o domiciliario no estaba controlado en absoluto.

Vinuesa Alvarez (1936), Inspector Provincial de Sanidad en Tarragona, o Molinero Manrique destacaban la importancia de los dispensarios antivenéreos en la lucha antileprosa, considerando además que se debía partir de un plan conjunto nacional con una organización centralizada; los esfuerzos locales no podían combatir la lepra extendida prácticamente por todo el territorio nacional (11).

En 1942 destaca la creación del Instituto Leprológico Nacional de Trillo, que pretendía ser el centrọ coordinador de investigaciones leprológicas en España, ademas de su labor de asistencia y aislamiento del leproso. Era de iniciativa pública y como su nombre indica tenia altas pretensiones: centralizar las medidas de lucha y las investigaciones tal y como se había recomendado repetidamente. En este mismo año se creó también el Consejo Nacional de Sanidad, dependiente de la Dirección General de Sanidad y constituido por once secciones: la sección I era la de Lepra y Enfermedades Sexuales. De nuevo se ligaban la lepra y las enfermedades venéreas, ambas pertenecientes al campo de la dermatología.

\subsection{Tercera etapa: de 1944 a 1953}

Es esta una etapa interesante en la lucha contra la lepra en España; varias razones podríamos aducir: de orden administrativo, avances de orden científico, avances terapéuticos, etc... Ya hablamos sobre la importancia tan decisiva que iba a tener la organización del VI Congreso Internacional de Leprología en Madrid en 1953. Cara a este congreso, los años anteriores se dedicaron a planificar una eficaz campaña contra la lepra. Las autoridades sanitarias tomaron por fin una parte más activa en este problema: José Antonio Palanca, Director General de Sanidad, fue uno de sus artífices.

Antonio Cordero Soroa fue puesto al frente de la lucha contra la lepra en diciembre de 1947: es el representante de la lucha oficial pero es bien acogido por la mayoría de los leprólogos y dermatólogos.

Los avances que se consiguieron fueron muchos; uno de ellos el gran impulso que se dio al censo de enfermos y convivientes. El número oficial de enfermos se incrementaba asombrosamente cada año, pero era por- 
que se iban censando los casos que anteriormente permanecían ocultos. Se empezó a contar también con censos de convivientes (12).

En una de las parcelas donde los descubrimientos fueron decisivos, fue en el tratamiento de la enfermedad. Por primera vez en la historia de la lepra se disponía de fármacos realmente eficaces, capaces de controlar la enfermedad en períodos de tiempo relativamente cortos y de esterilizar a los enfermos bacilíferos. Las sulfonas se introdujeron como arma terapéutica en España a partir de 1945 (13).

Las perspectivas de lucha cambiaron de manera importante, el poder controlar la enfermedad en un corto período de tiempo hizo que la reclusión no fuese ya la medida primordial y como consecuencia de ello, los sanatorios o leproserías tampoco iban a ser ya pilar fundamental en el control de la enfermedad.

Desde una perspectiva social, un punto de interés que trajo consigo el nuevo tratamiento fue que sacó, de forma indirecta, a la luz a muchos enfermos. Estos, para poder beneficiarse de la terapéutica salen de sus «escondrijos». Si antes se ocultaban para evitar ser recluidos o para evitar el rechazo social, ahora les interesa darse a conocer, con ello facilitan mucho la realización del censo, profilaxis y tratamiento (14).

Las bases legislativas que surgen en España durante estos años son entre otras: la Ley de Sanidad de 1944 que dedica su base . $^{\circ} 12$ a la «Organización contra la lepra y dermatosis», y que en conjunto insistía en los mismos puntos que la legislación anterior (1933). En 1949 a propuesta de la Comisión Permanente de la Sección de Lepra del Consejo Nacional de Sanidad se publicaron las Normas generales que rigen la profilaxis de la lepra en España; tiene 12 capítulos en los que se incluían las medidas siguientes:

I. Impedir la inmigración de nuevos casos.

II. Descubrimientos de casos autóctonos.

III. Formación del censo de enfermos y del censo de convivientes.

IV. Estudio epidemiológico de todos los casos censados.

V. Clasificación de los enfermos.

VI. Aislamiento obligatorio de todos los lepromatosos, de los indigentes y de aquellos otros que por su profesión o genero de vida resulten peligrosos para los demás.

VII. Separación obligatoria de los menores de los focos de contagio.

VIII. Tratamiento obligatorio de todos los enfermos de lepra, gratuito para los que no tengan medios de fortuna. 
IX. Vigilancia sanitaria de todos los enfermos, de sus convivientes y de los casos dudosos.

$\mathrm{X}$. Asistencia social a todos los enfermos y familiares, preferentemente a los niños.

XI. Educación sanitaria de los enfermos lazarinos, de sus convivientes y de cuantos rodean al enfermo.

XII. Preparación del personal técnico, organizando debidamente la propaganda y procurando lograr un perfeccionamiento de los médicos y auxiliares sanitarios (15).

Con estas disposiciones legales, unidas a los organismos oficiales de lucha, se tenían las bases para realizar la campaña antileprosa. Los sanitarios que la dirigen son los de la lucha antivenérea, dermatólogos oficiales del Estado. Esta campaña se hizo efectiva en la práctica en 1947-48, cuando ya se sabía que España iba a ser sede del VI Congreso Internacional de Leprología en 1953.

En diciembre de 1947, cuando Cordero Soroa se puso al frente de la campaña, lo primero que se propuso fue confeccionar un censo de enfermos y otro de convivientes (16). Otra cuestión primordial fue la organización de los Equipos Móviles, con un leprólogo al frente; su objetivo era revisar a los enfermos ya conocidos y descubrir nuevos casos entre los convivientes de las zonas de más alta endemia. Donde no había equipos móviles actuaban los Dispensarios Dermatológicos y de Higiene Social.

Según Cordero Soroa, es en ese momento cuando la lucha contra la lepra en España adquiere un auge importante; las razones que da él son: $10^{\circ}$ La labor de los especialistas haciendo campaña en pro de la lucha contra la lepra. $2 .^{\circ}$ El nuevo Estado que de verdad se ha enfrentado al problema de la lepra. $3 .^{\circ} \mathrm{El}$ hecho de que en el $V$ Congreso Internacional de Leprología celebrado en Cuba se determinase que el siguiente sería en Madrid:

"Lo que nos obliga a preparar una organización y perfeccionamiento de los servicios que nos permita presentarnos con el prestigio y decoro que la importancia del problema exige así como la valía de nuestros visitantes» (17).

La campaña antileprosa promovida por Cordero Soroa pudo por fin mostrar resultados eficaces en el VI Congreso Internacional de Leprología, 
celebrado en Madrid en 1953 (18). La lucha contra la lepra en España no había hecho más que comenzar.

\section{Conclusión}

Una vez realizado este breve análisis podemos afirmar que la lepra fue una enfermedad de gran transcendencia en España en la primera mitad del siglo XX y como consecuencia de ello se tomaron medidas para combatirla; estas medidas fueron evolucionando acordes a los avances. científicos. Las autoridades sanitarias tomaron parte activa en el problema de forma más o menos eficaz a lo largo de los años, y en todo caso hubo que vencer las barreras sociales que imponía el ser leproso.

\section{NOTAS}

(1) Granjel, L. (1986), La Medicina Española Contemporánea, Edic. Universidad de Salamanca, pp. 127-128.

(2) "Lucha contra la lepra en España» (1915). (Folleto de la Inspección General de Sanidad), Clínica Moderna, XIV, pp. 23-24. BeCARES, F. (1912), «Lepra, etiología, bacteriología, lucha antileprosa», Gaceta Médica Catalana, pp. 852, 447. GARCía DEL Mazo (1912), «Estado actual de la profilaxis de la lepra o cómo no se cumple una Real Orden», España Médica, 45, p. 12. Hauser, P. (1913), Geografía Médica de la Península Ibérica, tomo III, pp. 8-10.

(3) González Castellano, J. (1904), "De la lepra en España. Su profilaxis», Revista Española de Dermatología y Sifilografía, abril, pp. 146-150.

(4) Pérez Dagnino (1915), Concepto moderno de la lepra y su estado actual en la provincia de Alicante, tesis doctoral, p. 130. PANHON, F. (1904), "La lepra en España», Revista Española de Dermatología y Sifilografía, septiembre, pp. 533-535.

(5) Tello, J.F. (1909), «La lepra en España», II Conferencia Internacional de la lepra, p. 206.

(6) "Lucha contra la lepra en España», ibidem.

(7) SÁNCHez Covisa, J. (1928), El problema social de la lepra en España, Madrid, Establecimiento tipográfico, $58 \mathrm{pp}$. Con motivo de su ingreso en la Real Academia de Medicina pronunció este interesante discurso en el que aborda la problemática de la lepra en España desde diferentes ángulos.

(8) Bejarano, J. (1929), Profilaxis, tratamiento y estado actual de la lepra en España, Madrid, Javier Morata editor, pp. 30-32. 
(9) Orden Ministerial de 2 de septiembre de 1932 (1933), en Trabajos del Sanatorio de Fontilles.

(10) Montañés, P. (1934), «Proyecto de organización de la lucha contra la lepra en España», Ecos Españoles de Dermatología y Sifilografía, pp. 643-663. Comunicación al I Congreso Nacional de Sanidad.

(11) Vinuesa Alvarez (1937), La Profilaxis de la lepra y factores que la determinan, folleto, p. 42. VinUesa Alvarez (1938-39), «Esquema de una organización nacional antileprosa", Revista de Sanidad e Higiene Pública, p. 394. Molinero Manrique (1934) "Organización contra la lepra», I Congreso Nacional de Sanidad, p. 7.

(12) CORDERO SOROA (1953), "El problema de la lepra en España», VI Congreso Internacional de Leprologia, Madrid, p. 944. Al finalizar el año 1948 habia registrados 1.510 enfermos junto con 1.619 fichas de familiares con 6.344 convivientes. En 1952 ya se tenían registrados 2.728 enfermos, además de 313 casos que habían fallecido.

(13) Bourges (1968), Essai sur l'histoire de la lepre, Aubiere, Clermont Reproduction, p. 66.

(14) Garcí del Mazo (1919); "La lepra en España», La Medicina Social Española, IV, pp. 83-86.

(15) CORDERO SOROA (1953), op. cit. en nota 12, p. 971.

(16) CORDERO SOROA (1953), op. cit. en nota 12, pp. 923-976.

(17) CORdero Soroa (1948), "La lucha contra la lepra en España», Actas Dermosifilográficas, noviembre, p. 257.

(18) CORDERO SOROA (1953), «El problema de la lepra en España», VI Congreso Internacional de Leprología, Madrid, pp. 923-976. 